\title{
Modified flower petal technique in the treatment of Medina type $0,0,1$ or $0,1,0$ lesions
}

\author{
Murat Çayli*, MD; Zafer Elbasan, MD; Mustafa Gür, MD; Taner Şeker, MD; Hakan Uçar, MD; \\ Osman Kuloğlu, MD; Ömer Şen, MD; Durmuş Yıldıray Şahin, MD; Gülhan Yüksel Kalkan, MD \\ Department of Cardiology, Adana Numune Training and Research Hospital, Adana, Turkey
}

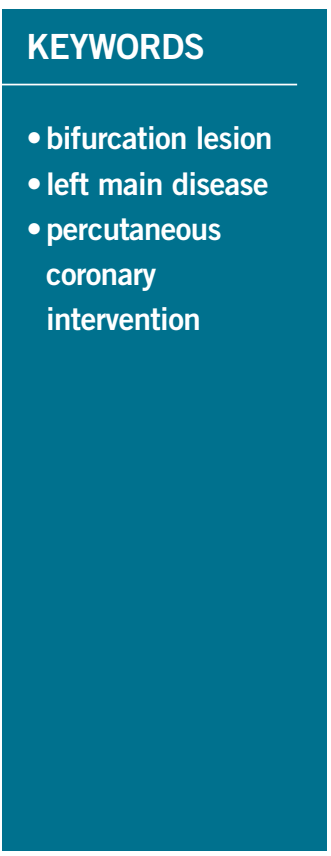

\begin{abstract}
Aims: The optimal strategy for patients with isolated ostial bifurcation lesions has not yet been determined. We propose the modified flower petal technique for the treatment of Medina type $0,0,1$ or $0,1,0$ coronary bifurcation lesions.
\end{abstract}

Methods and results: We selected 64 patients who had Medina type 0,0,1 or 0,1,0 coronary bifurcation lesions. Percutaneous coronary intervention (PCI) was performed with the modified flower petal technique in all patients. After PCI, all patients were followed up to nine months after the intervention. Quantitative coronary angiography (QCA) analyses were performed for both the main and the side branch at baseline, after the stent implantation and at nine-month follow-up. Twenty patients $(31.2 \%)$ had ostial left anterior descending artery lesions, nine patients $(14.1 \%)$ had ostial circumflex artery lesions and the other patients had isolated ostial non-left main bifurcation lesions. The procedural success rate was $100 \%$. There was no death, myocardial infarction, subacute or late stent thrombosis at nine-month follow-up. In one patient, instent restenosis requiring reintervention was noted.

Conclusions: The modified flower petal technique has excellent acute results and midterm clinical outcomes in the management of Medina type $0,0,1$ or $0,1,0$ coronary bifurcation lesions.

\footnotetext{
*Corresponding author: Department of Cardiology, Adana Numune Training and Research Hospital, Adana, Turkey. E-mail:drcayli@yahoo.com
} 


\section{Introduction}

Isolated ostial bifurcation lesions (Medina type $0,0,1$ or $0,1,0$ ) represent $<20 \%$ of coronary bifurcation lesions ${ }^{1,2}$. Percutaneous coronary intervention (PCI) of this subset of lesions is challenging for the interventional cardiologist because of the technical difficulty and potential risk of serious complications ${ }^{3}$. Stenting of such lesions may result in incomplete coverage of the ostial lesion or protrusion of the proximal stent margin into the adjacent vessel. Furthermore, potential plaque or carina shift during PCI may compromise the adjacent vessel ${ }^{3,4}$.

The optimal strategy for patients with isolated ostial bifurcation lesions has not yet been determined. Furthermore, Medina 0,0,1 lesions are usually managed medically because of the absence of reliable percutaneous treatment techniques ${ }^{3,5}$. Recently, we devised a new technique, called the modified flower petal technique, for the treatment of Medina type 1,1,1 coronary bifurcation lesions ${ }^{6}$, derived from flower petal stenting which was first defined by Kinoshita et $\mathrm{al}^{7}$. The most important characteristics of this technique are complete coverage of the side branch ostium by stent struts and a lower metallic burden at the carina region ${ }^{6}$. The aim of this study was to evaluate the technical feasibility, safety and short-term and midterm procedural and clinical results achieved with the modified flower petal technique for Medina type $0,0,1$ or $0,1,0$ coronary bifurcation lesions.

\section{Methods}

\section{PATIENT SELECTION}

Between April 2011 and June 2012, we included 64 patients (41 male, 23 female; mean age $60.0 \pm 11.2$ years) who had Medina type $0,0,1$ or $0,1,0$ coronary bifurcation lesions. The vessel size had to be $\geq 2.5 \mathrm{~mm}$ by visual estimation on coronary angiography. Patients with acute myocardial infarction (MI), in-stent restenotic lesions, heavily calcified lesions, severe proximal tortuosity, chronic total occlusion, cardiogenic shock or contraindications to prolonged use of antiplatelet agents were excluded. The local ethics committee approved the study.
Treatment options were fully discussed with the patient, and written informed consent for both the PCI procedure and participation in the study was obtained from all patients. All of the patients were brought back for angiography at nine months, which was considered to be part of the study method.

\section{PROCEDURE}

Previously, we have described the steps of this technique for the treatment of Medina 1,1,1 lesions ${ }^{6}$. The most important difference for Medina $0,0,1$ or $0,1,0$ is that a second stent was not implanted. The steps of this technique for Medina 0,1,0 or 0,0,1 lesions are illustrated in Figure 1 and Figure 2. A large (7 or 8 Fr) and strong back-up support guiding catheter must be selected for this technique. The procedure starts with wiring of both branches (the first wire into the target branch and second wire into the other branch as an anchor wire). A stent-balloon system is prepared outside of the guiding catheter. First, the plastic stent cover is pulled back slightly to expose the final proximal stent strut (Figure 1A), and the stent delivery system balloon is inflated to 5-6 atmospheres and deflated (Figure 1B). Then, the proximal end of the anchor wire is passed through the final proximal stent strut (Figure 1C). Another balloon is loaded on the anchor wire as an anchor balloon. Proximal markers of stent and balloon are aligned. Then the final proximal strut of the stent is re-crimped by hand (Figure 1D). In this article, one of the most important differences from our previous definition is the location of alignment of the stent and anchor balloon. The anchor balloon was centred on the last proximal stent strut in the previous article, whereas the proximal markers of the stent and the anchor balloon are positioned in the same line in this article. Thus, the stent-balloon system becomes more flexible and allows easy advancement of the stent or balloon into high take-off vessels.

The prepared stent-balloon system is advanced through the guiding catheter to the target lesion until the anchor balloon stops the advancement of the stent (Figure 2A). In this way, the anchor balloon can prevent excessive stent advancement into the target branch and provide complete coverage of the lesion by the stent struts.
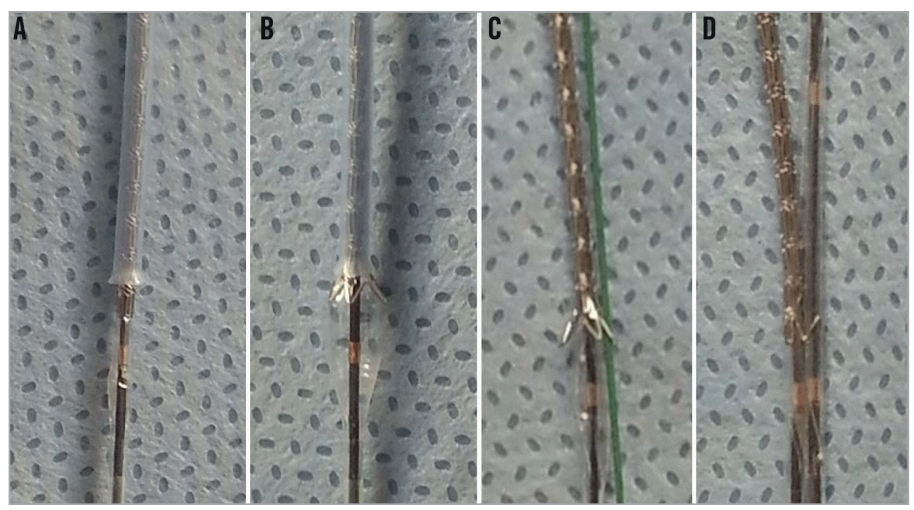

Figure 1. Preparation of the stent-balloon system. A) The plastic stent cover is pulled back slightly to expose the final proximal stent strut. B) The stent delivery system balloon is inflated to 5-6 atmospheres and deflated. C) The proximal end of the second wire is passed through the final proximal stent strut. D) Another balloon is loaded onto the second wire as an anchor balloon. The proximal markers of the stent and balloon are aligned. Then, the final proximal strut of the stent is re-crimped by hand. 


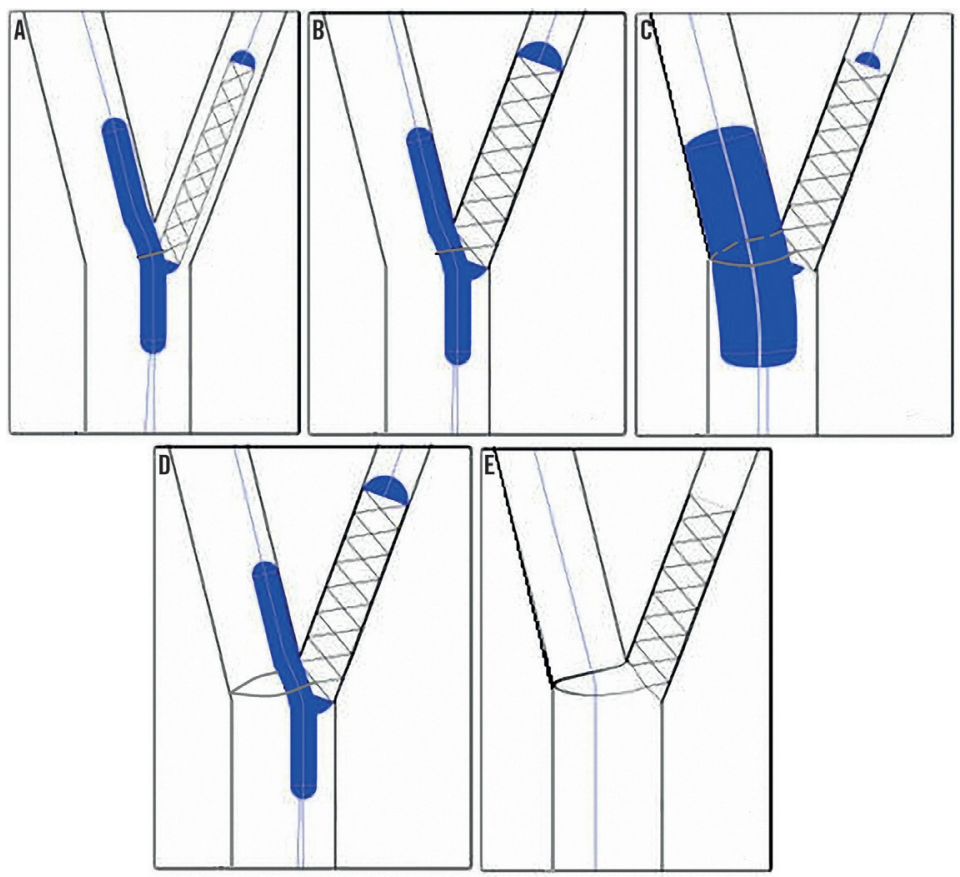

Figure 2. Schematic model presentation of the new technique. A) The prepared stent-balloon system is advanced through the guiding catheter to the lesion location until the anchor balloon stops the advancement of the stent. B) The stent balloon is inflated and deflated. C) The anchor balloon is inflated and deflated. D) The stent balloon is inflated again. E) The protruding final proximal strut comes into contact with the opposite side of the adjacent vessel wall.

First, the stent balloon is inflated and deflated (Figure 2B). Second, the anchor balloon is inflated and deflated (Figure 2C). Then, the stent balloon is inflated again (Figure 2D). Thus, the protruding final proximal strut is in contact with the opposite side of the adjacent vessel wall (Figure 2E). The most important characteristics of this technique are complete coverage of the ostial bifurcation lesion by stent struts without protruding into the adjacent vessel, and a lower metallic burden at the carina region (Figure 3, Figure 4, Figure 5).

\section{PREPARATIONS AND FOLLOW-UP}

All patients were pre-treated with aspirin $300 \mathrm{mg}$ and clopidogrel $300 \mathrm{mg}$ loading dose, followed by $75 \mathrm{mg}$ daily, at least one day prior to the index procedure. During the procedure, an intra-arterial bolus of unfractionated heparin was administered at a dosage of $70 \mathrm{U} / \mathrm{kg}$. All procedures were performed by using zotarolimus-eluting stents (Endeavor ${ }^{\circledR}$ Resolute; Medtronic, Minneapolis, MN, USA), sirolimus-eluting stents (Coracto ${ }^{\mathrm{TM}}$; Alvimedica, Istanbul, Turkey), paclitaxel-eluting stents (Coraxel; Alvimedica) and everolimus-eluting stents (XIENCE V ${ }^{\circledR}$; Abbott Vascular, Santa Clara, CA, USA). After the procedures, all patients were advised to stay on dual antiplatelet therapy for at least one year. All patients were followed up to nine months after the intervention. All patients adhered to dual antiplatelet therapy during the study period.

\section{DEFINITIONS AND CLINICAL ENDPOINTS}

Procedural success was defined as successful implantation of the stent into the target lesion, causing a final residual stenosis $\leq 30 \%$.
Major adverse cardiac events were defined as cardiac death, MI and target lesion revascularisation (TLR). TLR was defined as a repeat revascularisation with a stenosis $\geq 50 \%$ within the stent or in $5 \mathrm{~mm}$ proximal or distal segments adjacent to the stent. Stent thrombosis was defined as any of the following: angiographic demonstration of stent closure or intra-stent thrombosis, unexplained sudden death, or MI without concomitant documentation of a patent stent.

\section{QUANTITATIVE CORONARY ANGIOGRAPHY (QCA) ANALYSIS}

Quantitative coronary angiography analyses were performed at baseline, after stent implantation and at nine-month follow-up using dedicated bifurcation angiographic software (CardiOp-B system version 2.1.0.151; Paieon Medical, Ltd., Rosh Ha'ayin, Israel $)^{8,9}$. All analyses were performed in consensus by two experienced interventional cardiologists (M. Gür and Z. Elbasan) who were blinded to patient characteristics. QCA of each bifurcation lesion was obtained in three segments: the proximal main vessel, distal main branch and the side branch. For quantitative analysis, at least two orthogonal projections were obtained, and the angiographic frames with homogeneous contrast filling of the segment of interest were selected in a view offering good opening of the bifurcation. According to the algorithm in the dedicated software, reference vessel diameter, minimal lumen diameter (MLD) and diameter stenosis (DS) were measured in three segments. Binary restenosis was defined as $>50 \%$ diameter stenosis of the target lesion ${ }^{10}$. 

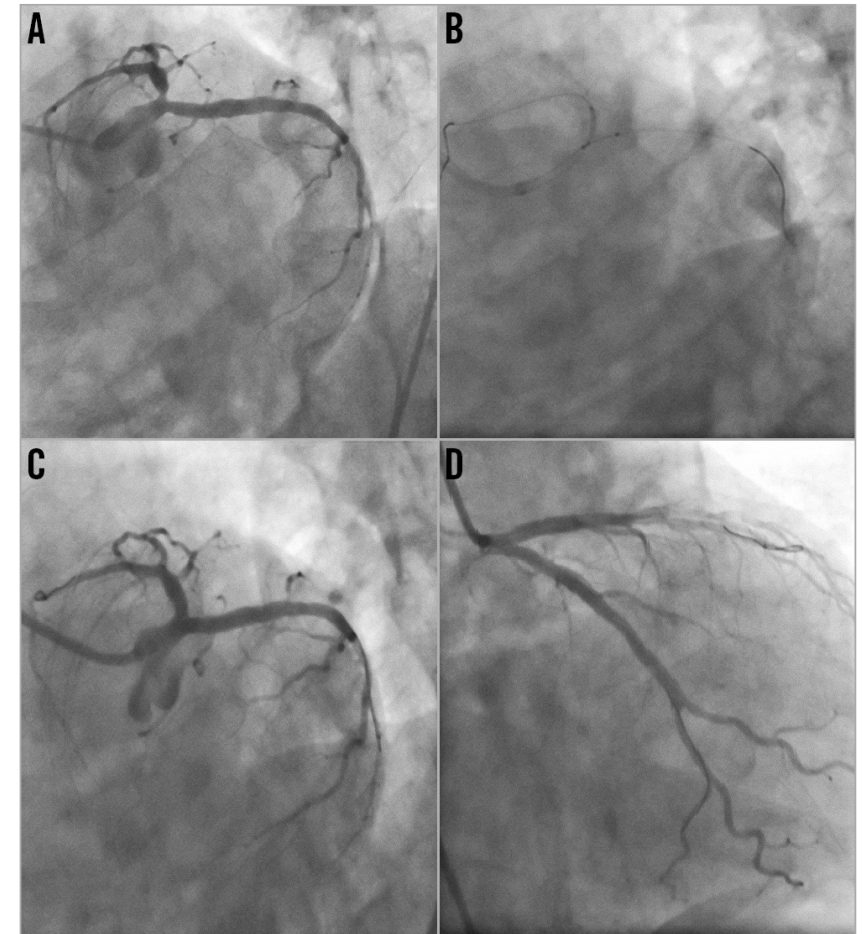

Figure 3. Stenting of isolated LAD ostial lesion with the modified flower petal technique. A) LAD ostial lesion viewed in $L A O-C A U$ projection. B) Positioning of the stent. $C \& D$ ) Angiographic results after stent implantation.

\section{STATISTICAL ANALYSIS}

All calculations were performed with Statistical Package for the Social Sciences, version 13.0 (SPSS Inc., Chicago, IL, USA). Continuous variables were expressed as mean \pm standard deviation, and categorical variables were presented as counts and percentages. A p-value $<0.05$ was considered significant.

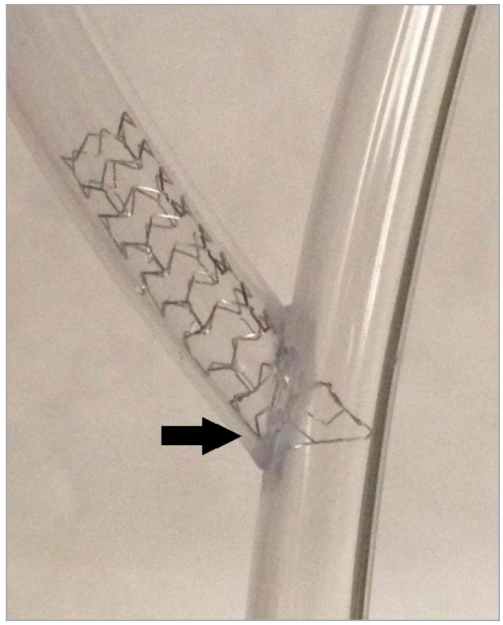

Figure 5. Bench testing of the modified flower petal technique for the treatment of an isolated ostial bifurcation lesion. The final position shows complete coverage of the ostial lesion by stent struts and minimal metallic burden at the carina region. There is no breakage or deformation on the stent.

\section{Results}

Table 1 shows the baseline clinical and angiographic characteristics of the patients. According to QCA analysis and visual estimation, 34 $(53.1 \%)$ and $30(46.9 \%)$ of the patients had Medina type $0,1,0$ and $0,0,1$, respectively. The mean age of patients was $60.0 \pm 11.2$ years, and $41(64.1 \%)$ patients were male. The most frequent PCI indication was stable angina $(82.8 \%)$. The lesion localisations were ostium of the left anterior descending (LAD) artery in $20(31.2 \%)$, mid level of the LAD artery in $14(21.9 \%)$, ostium of the circumflex artery in nine (14.1\%), ostium of the diagonal branch in $17(26.6 \%)$, and ostium of the obtuse marginal branch in four $(6.2 \%)$.

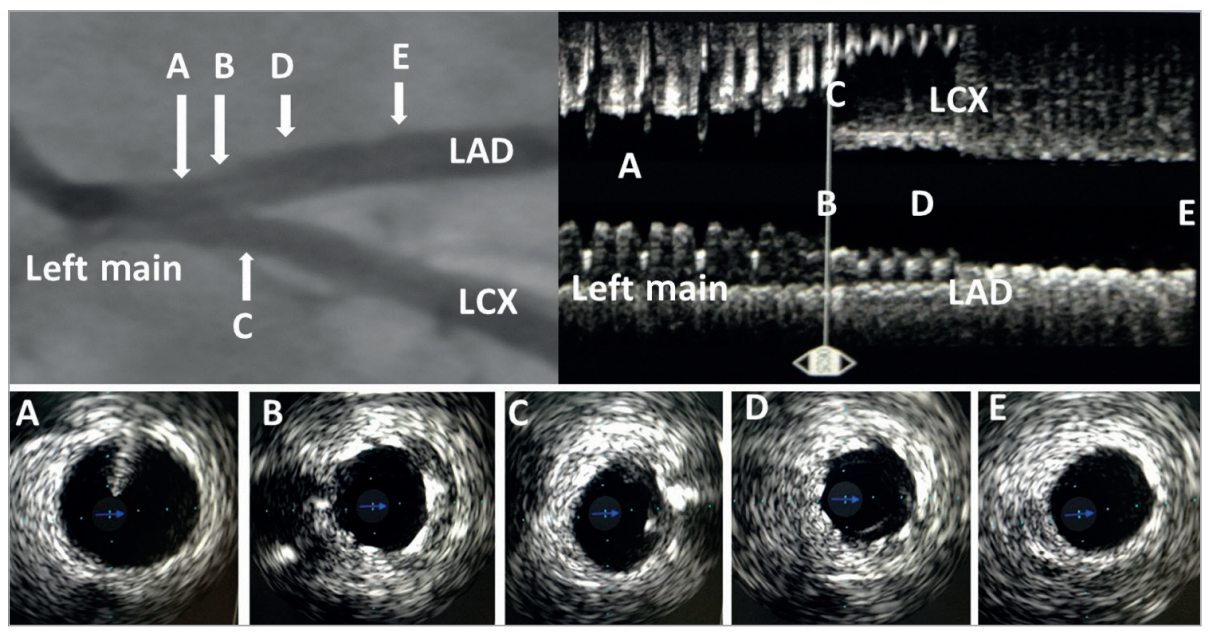

Figure 4. Angiographic image and IVUS findings after stenting of an isolated LAD ostial lesion with the modified flower petal technique. A) Cross-sectional intravascular image of the distal left main coronary artery. B) Cross-sectional intravascular image at the level of the carina shows complete coverage of the ostial lesion by the stent struts. After the procedure, the carina had a good position, without shifting to the LCX. C) Cross-sectional intravascular image of the LCX ostium. There was no plaque or carina shift into the LCX. D) Cross-sectional intravascular image shows well-apposed stent struts at the LAD proximal segment. E) Cross-sectional intravascular image of the LAD, just distal to the stenting area. LAD: left anterior descending artery; LCX: left circumflex artery 
Table 1. Baseline clinical and angiographic characteristics of patients $(n=64)$.

\begin{tabular}{|c|c|c|}
\hline \multicolumn{2}{|l|}{ Age, years } & \multirow{2}{*}{$\frac{60.0 \pm 11.2}{41(64.1)}$} \\
\hline Gender & Male, n (\%) & \\
\hline & Female, n (\%) & $23(35.9)$ \\
\hline \multicolumn{2}{|l|}{ Hypertension, n (\%) } & $27(42.2)$ \\
\hline \multicolumn{2}{|l|}{ Diabetes, $\mathrm{n}(\%)$} & $15(23.4)$ \\
\hline \multicolumn{2}{|c|}{ Hypercholesterolaemia, n (\%) } & $26(40.6)$ \\
\hline \multicolumn{2}{|l|}{ Smoking, n (\%) } & $26(40.6)$ \\
\hline \multicolumn{2}{|c|}{ Family history of coronary artery disease, $\mathrm{n}(\%)$} & $27(42.2)$ \\
\hline \multicolumn{2}{|c|}{ Prior myocardial infarction, $\mathrm{n}(\%)$} & $3(4.7)$ \\
\hline \multicolumn{2}{|l|}{ Prior $\mathrm{PCl}, \mathrm{n}(\%)$} & $4(6.3)$ \\
\hline \multicolumn{2}{|l|}{ Prior bypass surgery, n (\%) } & $0(0.0)$ \\
\hline \multirow[t]{2}{*}{$\mathrm{PCl}$ indication } & Unstable angina, $\mathrm{n}(\%)$ & $11(17.2)$ \\
\hline & Stable angina, $\mathrm{n}(\%)$ & $53(82.8)$ \\
\hline \multirow{2}{*}{$\begin{array}{l}\text { Bifurcation } \\
\text { characteristic, n (\%) }\end{array}$} & Medina $0,1,0$ & $34(53.1)$ \\
\hline & Medina $0,0,1$ & $30(46.9)$ \\
\hline \multirow{5}{*}{$\begin{array}{l}\text { Location of ostial lesion, } \\
\mathrm{n}(\%)\end{array}$} & LAD ostium & $20(31.2)$ \\
\hline & Circumflex ostium & $9(14.1)$ \\
\hline & LAD - mid level & $14(21.9)$ \\
\hline & Diagonal branch & $17(26.6)$ \\
\hline & Obtuse marginal branch & $4(6.2)$ \\
\hline
\end{tabular}

Table 2 shows procedural characteristics. Predilatation of the lesion was performed in 24 patients $(43.8 \%)$. The procedural success rate was $100 \%$. There was no major complication, but there were two minor intraprocedural complications, including dissection at the distal edge of the stent in two patients (managed by additional stent implantation). The anchor balloon did not prolapse into the target vessel in any patient. Because guidewires were twisted together, we could not advance the stent-balloon system to the lesion location in 10 patients $(15.6 \%)$. In these patients, advancement of the stent-balloon system was stopped, one of the wires was pulled back to the tip of the stent or balloon, the wires were untwisted, and the stent-balloon system was recrossed into the vessel. Then, the stent-balloon system could be successfully

Table 2. Procedural characteristics of patients $(n=64)$.

\begin{tabular}{|l|l|c|}
\hline Predilatation, $\mathrm{n}(\%)$ & $28(43.8)$ \\
\hline \multirow{2}{*}{$\begin{array}{l}\text { Type of stent, } \mathrm{n} \\
(\%)\end{array}$} & Zotarolimus-eluting stent & $11(17.2)$ \\
\cline { 2 - 3 } & Paclitaxel-eluting stent & $14(21.9)$ \\
\cline { 2 - 3 } & Sirolimus-eluting stent & $24(37.5)$ \\
\cline { 2 - 3 } & Everolimus-eluting stent & $15(23.4)$ \\
\hline Stent diameter, mm & $3.09 \pm 0.42$ \\
\hline Stent length, mm & $16.2 \pm 4.2$ \\
\hline Procedural success, $\mathrm{n}(\%)$ & $64(100.0)$ \\
\hline Final kissing balloon, $\mathrm{n}(\%)$ & $4(6.3)$ \\
\hline Periprocedural myocardial infarction, $\mathrm{n}(\%)$ & $0(0.0)$ \\
\hline Acute stent thrombosis, $\mathrm{n}(\%)$ & $0(0.0)$ \\
\hline In-hospital major adverse cardiac events, $\mathrm{n}(\%)$ & $0(0.0)$ \\
\hline
\end{tabular}

advanced to the lesion location in all patients. There was no major adverse cardiac event during hospital stay.

Clinical and angiographic follow-up was available in 59 patients (92.2\%). QCA analysis results for both the main and the side branch at baseline, after the procedure and at nine-month follow-up are summarised in Table 3. There was no cardiac death, MI, subacute

Table 3. Quantitative coronary angiographic analysis of patients.

\begin{tabular}{|c|c|c|c|}
\hline \multicolumn{2}{|c|}{ 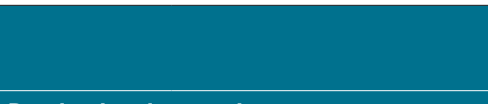 } & $\begin{array}{c}\text { Medina } 0,1,0 \\
(n=34)\end{array}$ & $\begin{array}{c}\text { Medina } 0,0,1 \\
(n=30)\end{array}$ \\
\hline \multicolumn{4}{|c|}{ Proximal main vessel } \\
\hline \multirow[t]{4}{*}{ Baseline } & RVD, $\mathrm{mm}$ & $3.42 \pm 0.43$ & $3.39 \pm 0.37$ \\
\hline & MLD, mm & $3.03 \pm 0.39$ & $2.98 \pm 0.37$ \\
\hline & Diameter stenosis, \% & $11.4 \pm 3.2$ & $12.1 \pm 3.5$ \\
\hline & Binary stenosis, n (\%) & $0(0.0)$ & $0(0.0)$ \\
\hline \multirow[t]{4}{*}{ Post procedure } & RVD, mm & $3.43 \pm 0.39$ & $3.40 \pm 0.40$ \\
\hline & MLD, mm & $3.05 \pm 0.41$ & $3.01 \pm 0.39$ \\
\hline & Residual stenosis, \% & $11.1 \pm 3.5$ & $11.5 \pm 4.0$ \\
\hline & Binary residual stenosis, $\mathrm{n}(\%)$ & $0(0.0)$ & $0(0.0)$ \\
\hline \multirow[t]{5}{*}{ Follow-up } & RVD, mm & $3.39 \pm 0.37$ & $3.36 \pm 0.38$ \\
\hline & MLD, mm & $2.96 \pm 0.31$ & $2.92 \pm 0.43$ \\
\hline & Diameter of restenosis, $\%$ & $12.7 \pm 3.9$ & $13.1 \pm 3.1$ \\
\hline & Late lumen loss, mm & $0.09 \pm 0.04$ & $0.09 \pm 0.03$ \\
\hline & Binary restenosis, $n(\%)$ & $0(0.0)$ & $0(0.0)$ \\
\hline \multicolumn{4}{|c|}{ Distal main branch } \\
\hline \multirow[t]{4}{*}{ Baseline } & RVD, mm & $3.19 \pm 0.37$ & $3.15 \pm 0.37$ \\
\hline & MLD, mm & $0.71 \pm 0.43$ & $2.60 \pm 0.43$ \\
\hline & Diameter stenosis, \% & $77.4 \pm 9.5$ & $17.5 \pm 3.4$ \\
\hline & Binary stenosis, n (\%) & $34(100.0)$ & $0(0.0)$ \\
\hline \multirow[t]{4}{*}{ Post procedure } & RVD, $\mathrm{mm}$ & $3.21 \pm 0.39$ & $3.07 \pm 0.41$ \\
\hline & MLD, mm & $2.90 \pm 0.34$ & $2.62 \pm 0.45$ \\
\hline & Residual stenosis, \% & $9.7 \pm 0.4$ & $14.9 \pm 4.5$ \\
\hline & Binary residual stenosis, $\mathrm{n}(\%)$ & $0(0.0)$ & $0(0.0)$ \\
\hline \multirow[t]{5}{*}{ Follow-up } & RVD, mm & $3.17 \pm 0.40$ & $3.11 \pm 0.40$ \\
\hline & MLD, mm & $2.76 \pm 0.31$ & $2.44 \pm 0.41$ \\
\hline & Diameter of restenosis, \% & $12.9 \pm 0.3$ & $21.5 \pm 3.6$ \\
\hline & Late lumen loss, mm & $0.14 \pm 0.05$ & $0.18 \pm 0.04$ \\
\hline & Binary restenosis, $n(\%)$ & $0(0.0)$ & $0(0.0)$ \\
\hline
\end{tabular}

Side branch

\begin{tabular}{|l|l|c|c|}
\hline Baseline & RVD, $\mathrm{mm}$ & $2.79 \pm 0.42$ & $2.71 \pm 0.37$ \\
\cline { 2 - 4 } & MLD, $\mathrm{mm}$ & $2.34 \pm 0.53$ & $0.61 \pm 0.51$ \\
\cline { 2 - 4 } & Diameter stenosis, \% & $16.1 \pm 3.5$ & $77.5 \pm 9.9$ \\
\cline { 2 - 4 } & Binary stenosis, $\mathrm{n}(\%)$ & $0(0.0)$ & $30(0.0)$ \\
\hline \multirow{5}{*}{ Post procedure } & RVD, $\mathrm{mm}$ & $2.75 \pm 0.39$ & $2.74 \pm 0.43$ \\
\cline { 2 - 4 } & MLD, $\mathrm{mm}$ & $2.32 \pm 0.72$ & $2.46 \pm 0.66$ \\
\cline { 2 - 4 } & Residual stenosis, \% & $15.6 \pm 2.8$ & $10.3 \pm 0.6$ \\
\cline { 2 - 4 } Follow-up & Binary residual stenosis, $\mathrm{n}(\%)$ & $0(0.0)$ & $0(0.0)$ \\
\cline { 2 - 4 } & RVD, $\mathrm{mm}$ & $2.76 \pm 0.37$ & $2.72 \pm 0.41$ \\
\cline { 2 - 4 } & MLD, $\mathrm{mm}$ & $2.11 \pm 0.4$ & $1.75 \pm 1.13$ \\
\cline { 2 - 4 } & Diameter stenosis, \% & $23.6 \pm 4.9$ & $35.7 \pm 16.6$ \\
\cline { 2 - 4 } & Late lumen loss, $\mathrm{mm}$ & $0.21 \pm 0.04$ & $0.71 \pm 0.42$ \\
\cline { 2 - 4 } & Binary restenosis, $\mathrm{n}(\%)$ & $0(0.0)$ & $1(3.3)$ \\
\hline
\end{tabular}

MLD: minimum lumen diameter; RVD: reference vessel diameter 
or late stent thrombosis at nine-month follow-up. Only one patient $(1.7 \%)$ had binary restenosis as shown by QCA. This patient also had exertional angina and therefore we performed only balloon angioplasty due to focal in-stent restenosis. Thus, only one patient showed a major adverse cardiac event and TLR by PCI (Table 4).

Table 4. Clinical follow-up at 9 months $(n=59)$.

\begin{tabular}{|l|l|}
\hline Cardiac death, $\mathrm{n}(\%)$ & $0(0.0)$ \\
\hline Myocardial infarction & $0(0.0)$ \\
\hline Subacute stent thrombosis & $0(0.0)$ \\
\hline Late stent thrombosis & $0(0.0)$ \\
\hline Target lesion revascularisation & $1(1.7)$ \\
\hline Cumulative & $1(1.7)$ \\
\hline
\end{tabular}

\section{Discussion}

The most important characteristics of the modified flower petal technique in patients with Medina $0,1,0$ or $0,0,1$ lesions are complete coverage of the ostial bifurcation lesion by the stent struts without protruding into the adjacent vessel, a lower metallic burden at the carina region and easy application. This study showed that the modified flower petal technique also offered high immediate procedural success and excellent nine-month outcome in patients with ostial bifurcation lesions.

\section{ISOLATED LAD OR CIRCUMFLEX ARTERY OSTIAL LESIONS}

Isolated LAD or circumflex artery ostial lesions are considered to be unfavourable for percutaneous treatment because of the technical difficulty and potential risks. There are no specific recommendations for PCI in isolated circumflex ostial lesions ${ }^{3,11}$. However, two percutaneous treatment strategies are traditionally recommended in isolated LAD ostial lesions: floating stenting ${ }^{11}$ (precise stent implantation at the LAD ostium level with minimal protrusion into the area of the carina), and the crossover technique ${ }^{12}$ (a single stent is deployed from the distal left main vessel into the LAD crossing over the circumflex artery).

Medina et $\mathrm{al}^{11}$ recommended the implantation of a floating stent under angiographic/intravascular ultrasound (IVUS) guidance in the percutaneous treatment of ostial LAD lesions, without disease at the level of the distal left main coronary artery (LMCA) and circumflex origin. With this technique, angiographic compromise of the circumflex ostium was reported in 19 patients $(26 \%)$, but was significant in only seven patients $(10 \%)$. They concluded that carina displacement was the main mechanism of compromise of the circumflex ostium ${ }^{11}$. The European Bifurcation Club reported that, in cases with large bifurcation angles and IVUS documentation of the absence of disease in the distal LMCA, the floating stent technique is acceptable for the percutaneous treatment of isolated ostial LAD lesions ${ }^{3}$. However, optimal positioning of stenting in this technique is difficult. If the stent is positioned too proximally, it protrudes into the LMCA: this may compromise the circumflex ostium. Conversely, if the stent is positioned too distally, it does not entirely cover the ostial LAD lesion, which may result in acute recoil and late restenosis. Furthermore, stent positioning may be affected by cardiac movements. Therefore, optimal positioning of the stents is very important in this technique. For determining optimal stent positioning, Medina et $\mathrm{al}^{11}$ used IVUS guidance in $70 \%$ of cases. In the modified flower petal technique, the stent and anchor balloon are advanced together to the lesion location until the anchor balloon stops the advancement of the stent (Figure 2A). In this way, the anchor balloon can prevent excessive stent advancement into the target vessel and provide complete coverage of the lesion by stent struts without any manipulation. In our technique, inflation of the anchor balloon at nominal pressure into the adjacent vessel facilitates damage correction through repositioning of the carina.

Cubeddu et $\mathrm{al}^{12}$ proposed the crossover technique as the treatment approach for isolated ostial LAD lesions due to the potential risk of not totally covering the lesion. In this technique, the stent is placed from the distal LMCA to the LAD by crossing the circumflex artery ostium followed by final kissing balloon inflation (FKBI) to correct the carina position. However, this technique has some disadvantages, such as potential incomplete apposition of the stent in the LMCA due to the different diameters of the LMCA and side branch, the requirement of kissing balloon inflation, the use of longer stent lengths and the presence of metal in the LMCA. Furthermore, in an IVUS study, Medina et a ${ }^{11}$ reported that plaque was not found at the level of the carina in most patients (73\%) with isolated LAD ostial lesions. We showed that the modified flower petal technique avoids metallic burden in a disease-free segment such as the LMCA, provides optimal positioning of the stent in the ostium of the LAD/circumflex artery, and does not require routine FKBI to correct the carina position.

\section{ISOLATED OSTIAL NON-LEFT MAIN BIFURCATION LESIONS}

Isolated side branch ostial lesions (Medina type 0,0,1) represent $<5 \%$ of coronary bifurcation lesions and are very challenging lesions (especially in a Y-type bifurcation angle) $)^{1,13}$. The technical challenges are the problem of perfect ostial stent positioning, stent protrusion into the main vessel, and/or poor ostial coverage of the side branch lesion and high risk of plaque or carina shift (in the main vessel) $)^{3,4}$. The optimal strategy for patients with isolated ostial side branch lesions has not yet been determined ${ }^{3}$. A risk of injury to the main vessel when treating the side branch exists with all PCI techniques. For this reason, this subset of bifurcation lesions is usually managed medically ${ }^{5}$.

Several techniques, such as placement of a non-inflated balloon in the main vessel ${ }^{3,14}$ or the Szabo technique ${ }^{15}$, have been described to enhance the accuracy of stent placement. However, despite the use of these techniques, percutaneous treatment of ostial side branch lesions has an increased risk of incomplete coverage of the side branch ostium or stent protrusion into the main vessel, especially in a narrow bifurcation angle ${ }^{13,16}$. To overcome these problems, Brunel et $\mathrm{al}^{13}$ proposed the inverted provisional technique, with placement of the stent from the proximal main vessel into the side branch and final kissing, offering the advantage of 
complete coverage of the ostium. Complete coverage of the ostial side branch is obtained in all cases with this technique. However, this technique has some disadvantages, such as incomplete apposition of the stent in the proximal main vessel due to the different diameters of the proximal main vessel and side branch, the requirement of kissing balloon, the use of longer stent lengths and the presence of metal in the main vessel. Furthermore, strut crossing failure with this technique may compromise the main vessel. In a recently published, small study, Grundeken et al ${ }^{16}$ successfully treated 12 patients with Medina 0,0,1 lesions using a dedicated side branch stent alone (Tryton Side Branch Stent; Tryton Medical, Durham, NC, USA). This is a modification of the inverted provisional technique (modified Brunel technique). They reported $100 \%$ procedural success and acceptable long-term results. The most important disadvantage of this technique is the lack of drug on the bare metal Tryton, potentially resulting in an increase of the restenosis rate. Other potential disadvantages of the modified Brunel technique are insufficient radial strength and incomplete apposition of the wedding bands in the proximal part of the stent and the three panels of the transition zone ${ }^{16}$.

Szabo et a ${ }^{15}$ described a bifurcation technique for the treatment of isolated ostial bifurcation lesions by using the target wire and the anchor wire. In this technique, the anchor wire which passes through the most proximal stent strut helps to prevent excessive progression of the stent beyond the ostium and facilitates the precise stenting of the ostium. Recently, Gutiérrez-Chico et al ${ }^{17}$ reported that the Szabo technique is more accurate than the conventional technique in avoiding malpositioning of the stent. However, the Szabo technique is not the ideal technique due to technical difficulties. The anchor wire can be prolapsed into the target branch and the stent can advance excessively into the target branch, leading to incomplete coverage of the target branch ostium in the Szabo technique ${ }^{18}$. In our study, the anchor balloon did not prolapse into the target vessel in any patient. Because the anchor wire and the anchor balloon together provide stronger support, they do not prolapse into the target branch in the modified flower petal technique. In this way, the modified flower petal technique provides complete coverage of the target branch ostium. The most important difference between the modified flower petal technique and the Szabo technique is the expansion of the most proximal stent cell to the opposite vessel wall in the main branch. Therefore, in contrast to the Szabo technique, there is no protrusion of the stent strut into the main lumen in the modified flower petal technique. Furthermore, we think that due to the stent and anchor balloon being advanced together, the modified flower petal (MFP) technique has increased pushability compared to the Szabo technique.

Today, there is no consensus regarding the best technical approach for Medina 0,0,1 lesions ${ }^{3}$. We propose the modified flower petal technique for the treatment of this subset of bifurcation lesions. In this technique, the guidewire and balloon which are placed into the main vessel restrain the further advancement of the side branch stent, leading to complete coverage of the side branch ostium without protrusion of the stent into the main vessel. Unlike previous techniques, this technique provides complete coverage of the side branch ostium and does not require routine FKBI, and the presence of metal in the main vessel is minimal.

\section{Limitations}

This study is a single-centre experience with a relatively small sample size. This technique was not compared with other techniques such as floating stenting, the crossover technique, the Szabo technique or the inverted provisional technique. However, this is the first study to have evaluated the technical feasibility, safety and immediate and midterm procedural and clinical results achieved with the modified flower petal technique for Medina type $0,0,1$ or $0,1,0$ coronary bifurcation lesions. After these encouraging results, further larger randomised studies with longer follow-up, including comparing our technique with other techniques, are needed. In this study, twisting of wires was noted in only 10 patients $(15.6 \%)$. However, this situation did not affect the success of the procedure. Another important disadvantage of our technique is decreased flexibility of the stent during advancement of the stent-balloon system. For this reason, this technique is not suitable for tortuous lesions. Intravascular ultrasound, or even better, optical coherence tomography, would certainly have been able to assess this result more accurately, but these were not performed systematically.

\section{Conclusions}

This study showed that the modified flower petal technique has excellent acute results and midterm clinical outcomes in the management of Medina type $0,0,1$ or $0,1,0$ coronary bifurcation lesions. It also has a number of advantages, such as complete lesion coverage while protecting the adjacent vessel, and a lower metallic burden at the carina region. However, additional randomised studies with larger sample sizes and longer follow-up are necessary to establish the long-term safety and efficacy of this technique.

\section{Impact on daily practice}

PCI of isolated ostial bifurcation lesions is challenging and associated with higher procedural and medium-term complication rates. The optimal strategy for patients with isolated ostial bifurcation lesions has not yet been determined. We propose a new technique, the "modified flower petal technique", for the treatment of this subset of bifurcation lesions. Unlike previous techniques, this technique is easy to apply, provides complete coverage of the side branch ostium and does not require routine FKBI.

\section{Conflict of interest statement}

The authors have no conflicts of interest to declare.

\section{References}

1. Ferenc M, Gick M, Kienzle RP, Bestehorn HP, Werner KD, Comberg T, Kuebler P, Büttner HJ, Neumann FJ. Randomized trial 
on routine vs. provisional T-stenting in the treatment of de novo coronary bifurcation lesions. Eur Heart J. 2008;29:2859-67.

2. Adriaenssens T, Byrne RA, Dibra A, Iijima R, Mehilli J, Bruskina O, Schömig A, Kastrati A. Culotte stenting technique in coronary bifurcation disease: angiographic follow-up using dedicated quantitative coronary angiographic analysis and 12-month clinical outcomes. Eur Heart J. 2008;29:2868-76.

3. Stankovic G, Darremont O, Ferenc M, Hildick-Smith D, Louvard Y, Albiero R, Pan M, Lassen JF, Lefèvre T; European Bifurcation Club. Percutaneous coronary intervention for bifurcation lesions: 2008 consensus document from the fourth meeting of the European Bifurcation Club. EuroIntervention. 2009;5: 39-49.

4. Iakovou I, Ge L, Colombo A. Contemporary stent treatment of coronary bifurcations. J Am Coll Cardiol. 2005;46:1446-55.

5. Brueck M, Heidt M, Kramer W, Ludwig J. Comparison of interventional versus conservative treatment of isolated ostial lesions of coronary diagonal branch arteries. Am J Cardiol. 2004;93:1162-4.

6. Çaylı M, Şahin DY, Elbasan Z, Gür M, Çağlıyan CE, Kıvrak A, Şeker T, Ballı M. Modified flower petal technique: a new technique for the treatment of Medina type 1.1.1 coronary bifurcation lesions. JACC Cardiovasc Interv. 2013;6:516-22.

7. Kinoshita Y, Katoh O, Matsubara T, Ehara M, Nasu K, Habara M, Suzuki T. First clinical experience of "flower petal stenting": a novel technique for the treatment of coronary bifurcation lesions. JACC Cardiovasc Interv. 2010;3:58-65.

8. Schlundt C, Kreft JG, Fuchs F, Achenbach S, Daniel WG, Ludwig J. Three-dimensional on-line reconstruction of coronary bifurcated lesions to optimize side-branch stenting. Catheter Cardiovasc Interv. 2006;68:249-53.

9. Gollapudi RR, Valencia R, Lee SS, Wong GB, Teirstein PS, Price MJ. Utility of three-dimensional reconstruction of coronary angiography to guide percutaneous coronary intervention. Catheter Cardiovasc Interv. 2007;69:479-82.

10. Ormiston JA, Webster MW, El Jack S, Ruygrok PN, Stewart JT, Scott D, Currie E, Panther MJ, Shaw B, O’Shaughnessy B.
Drug-eluting stents for coronary bifurcations: bench testing of provisional side-branch strategies. Catheter Cardiovasc Interv. 2006;67:49-55.

11. Medina A, Martín P, Suárez de Lezo J, Amador C, Suárez de Lezo J, Pan M, Melián F, Hernández E, Burgos L, Ojeda S, Ortega JR, García A. Vulnerable carina anatomy and ostial lesions in the left anterior descending coronary artery after floating-stent treatment. Rev Esp Cardiol. 2009;62:1240-9.

12. Cubeddu RJ, Wood FO, Saylors EK, Mann T. Isolated disease of the ostium left anterior descending or circumflex artery: management using a left main stenting technique. Clinical outcome at 2 years. J Invasive Cardiol. 2007;19:457-61.

13. Brunel P, Martin G, Bressollette E, Leurent B, Banus Y. "Inverted" provisional T stenting, a new technique for Medina 0,0,1 coronary bifurcation lesions: feasibility and follow-up. EuroIntervention. 2010;5:814-20.

14. Mishell JM, Vakharia KT, Ports TA, Yeghiazarians Y, Michaels AD. Determination of adequate coronary stent expansion using StentBoost, a novel fluoroscopic image processing technique. Catheter Cardiovasc Interv. 2007;69:84-93.

15. Kern MJ, Ouellette D, Frianeza T. A new technique to anchor stents for exact placement in ostial stenoses: the stent tail wire or Szabo technique. Catheter Cardiovasc Interv. 2006;68:901-6.

16. Grundeken MJ, Agostoni P, Lesiak M, Koch KT, Voskuil M, de Winter RJ, Wykrzykowska JJ, Stella PR. Placement of Tryton Side Branch Stent only; a new treatment strategy for Medina 0,0,1 coronary bifurcation lesions. Catheter Cardiovasc Interv. 2013;82: E395-402.

17. Gutiérrez-Chico JL, Villanueva-Benito I, VillanuevaMontoto L, Vázquez-Fernández S, Kleinecke C, Gielen S, IñiguezRomo A. Szabo technique versus conventional angiographic placement in bifurcations 010-001 of Medina and in aorto-ostial stenting: angiographic and procedural results. EuroIntervention. 2010;5:801-8.

18. Jain RK, Padmanabhan TN, Chitnis N. Causes of failure with Szabo technique - an analysis of nine cases. Indian Heart J. 2013;65:264-8. 